\title{
Nuclear Physics in Europe Planning The Future
}

\author{
Ingo Sick \\ Institute for Physics, University of Basel, Switzerland
}

\begin{abstract}
The larger and more complex facilities needed to ensure leadership in nuclear physics will call for greater cooperation at the European level starting at the initial planning stages.
\end{abstract}

Research in fundamental nuclear physics was done in the past at the national level, with accelerators and equipment that were small enough so that many countries could exploit a fair fraction of the complement of facilities needed by a research programme having the breadth required by nuclear physics.

This scene is rapidly changing. International collaboration, and European collaboration in particular, is increasing. A number of existing facilities in Europe are one of a kind and are already exploited by a sizeable international community. But this collaboration at present still takes place largely on an informal basis.

When considering plans for the future, it is clear that this mode of operation is likely to change. While small, university-based facilities — important for the education of students - will continue to operate according to purely national criteria, the future large research institutions of nuclear physics will be of European scale. They will be planned, built and exploited by a European community of physicists. This need for closer European cooperation will have an impact on the planning of future tools for research: the present report describes some of the recent developments in this direction.

\section{National Long-Range Plans}

Detailed long-range plans for nuclear physics are available for a number of

Professor Ingo Sick has held the chair of experimental nuclear physics in the Institute for Physics, University of Basel, Klingelbergstrasse $82, \mathrm{CH}-4056 \mathrm{Basel}$ since 1983. $\mathrm{He}$ received his Ph.D. in 1967 from the University, where he became a group leader after working as a postdoc at Stanford University, USA and as a Research Associate at CEN, Saclay. Awarded the Bonner Prize of the American Physical Society in 1987, he became doctor honoris causa of the University of Utrecht in 1988.
European countries. They give a good idea where the individual communities are heading. These plans in general document a fair awareness of developments in other countries, but they rarely show the strong correlations needed in the future.

\section{France}

The most recent 'look into the future' is the one of France. Contrary to standard planning documents, which in general are produced by the physicists themselves who are involved this report is more in the style of an evaluation by outsiders. The document (1), dealing with both the current status and future trends, was elaborated by a group with mainly particle physics backgrounds from the French Académie des Sciences. These special features make the report particularly interesting so we shall discuss it in more detail.

The report identifies a number of general trends that characterize the development of nuclear physics. It notes a certain convergence of particle and nuclear physics, both in terms of the theme - the study of QCD and its consequences for the understanding of the strong interaction and nuclei; and tools - the use of large accelerators of multinational scale. The latter aspect leads to a growing international character.

The report also notes a transition of the emphasis in nuclear physics. While much of the research in the past was devoted to the structure of individual quantum states of nuclei, the future will see increasing use of the nucleus as 'hadronic matter'. This evolution occurs in fields like heavy-ion reactions where the macroscopic properties of 'nuclear matter' are studied. It also occurs in the investigation of the nucleus with leptonic probes, where the interaction of the quark - the primary constituent hit by the high-energy lepton - with nuclear matter is becoming a topic of principal interest.
The report of the Académie des Sciences delineates a number of different areas of nuclear physics, with different prospects for the future:

- Nuclear structure physics aiming at the investigation of the properties of individual states. This area of research has been the main focal point in the past, and continues to produce results of high interest. To cite only a few, let us mention the superdeformed nuclei with extremely high angular momenta $(60 \%)$ produced in heavy-ion collisions (see Europhys. News 21 (1990) 86), or the exotic nuclei far from the line of stability produced by spallation at facilities such as ISOLDE and GANIL. These types of research will continue, but the report estimates that they will capture interest in the intermediate range (5-10 years).

- The understanding of the macroscopic properties of nuclei in collision. This direction of research has gained increasing prominence with the use of the many medium energy (tens of MeV per nucleon) heavy ion accelerators that are now available. The dynamics of nucleus-nucleus collisions, the fragmentation of nuclei, and the nuclear equation of state at low temperature are the main foci. The report estimates that this type of research remains interesting for an intermediate period only. The main emphasis is foreseen to shift to higher energies where higher densities and temperatures can be reached. It is suggested that this type of research take place at the new SIS facility in Darmstadt ( $2 \mathrm{GeV}$ per nucleon), where radioactive beams will be available as well. - Nuclear matter under extreme conditions. This area represents one of the main directions for the future. Contrary to the above-mentioned fields which deal with the nucleonic degrees of freedom of nuclei only, it aims at the study of phenomena related to the composite nature of nucleons. By studying nuclear matter at extremely high density and temperature, the phase transition from 
nucleons to quarks and gluons can be reached. This area of research is seen in the report as a very promising, longterm programme. It has already started at CERN with the beams of relativistic light ions that are now available, and will continue with the lead beams of $\geq 10$ $\mathrm{GeV}$ per nucleon (in the centre-of-mass system) which will hopefully be available soon (see Europhys. News 19 (1988) 133). The long-term prospects, beyond the ones related to the RHIC accelerator in the USA, could be TeV per nucleon heavy ion collisions in a future CERN hadron collider - the LHC.

- Subnucleonic degrees of freedom of nuclei. This topic has increasingly been the focus of recent experimental work with light hadronic and electromagnetic probes. Observations such as the celebrated EMC effect (the change of quark momentum distribution due to nuclear binding - see Europhys. News 19 (1988) 129), the properties of nucleonic resonances in nuclei, and understanding of the meson exchange currents observed in electron-nucleus scattering, have captured widespread interest.

For hadronic probes, the report recognizes the great variety of topics studied, but it also notes that a long-term future depends on the availability of higher energy projectiles; the future of this field thus is linked to high energy facilities such as KAON proposed in Canada.

It is for the electromagnetic probe where the report from the Académie des Sciences sees the greatest potential for future development, and a longrange programme of exciting science. The combination of the well-understood nature of the probe, and the potential of reaching very high spatial resolutions, make leptons particularly promising as tools to study hadronic systems down to a distance scale of $0.1 \mathrm{fm}$, where quark and gluon degrees of freedom dominate. Electron beams with energies of $>10 \mathrm{GeV}$ per beam would allow one to investigate the transition from the confinement dominated regime - the main one of interest for the study of QCD - to the scaling region where a theoretical description in terms of weakly interacting quarks and gluons is under fairly good control.

Such high energy, continuous electron beams of high intensity are today not available anywhere. Needless to say, the appropriate accelerator would be a project of European scale, and its optimised exploitation needs a constituency of physicists of European size.

\section{Other countries}

As pointed out above, we have descri- bed the French report in some detail given the special nature of this 'outside evaluation'. The planning documents of other European countries (as far as they are available) can only be discussed summarily in order to show both the similarities and the differences that often reflect national traditions and special situations.

Germany has produced a very detailed and in-depth analysis of the future of its nuclear physics research. A report (2) was published in 1988 by a panel assembled by the Bundesministerium für Forschung und Technologie (BMFT). In many respects this report comes to a similar set of priorities in discussing the physics addressed above in the context of the French report. It makes similar observations concerning general trends in the field, internationalization, the use of large facilities, and the closing of the gap between particle and nuclear physics in both physics topics and experimental technology. To avoid duplication, we shall not repeat the analysis of future developments of the physics, but only mention some of the more practical consequences that concern the rôles envisaged for the various facilities.

The German report sees a decreasing interest in the research with heavy ion beams of intermediate energies. As for the French report, it identifies heavy ion physics at the higher energies as a main priority, and sees much of the research moving to the new SIS/ESR facility in Darmstadt. For experiments aiming ultimately at the quark-gluon plasma, the main activity is expected to take place at CERN with the beams of relativistic heavy ions.

Research with hadronic probes is also envisaged to be most interesting at the higher energy covered by KAON; for the lower energies ( $\leq 2 \mathrm{GeV}$ ) a number of special topics can be studied with the German COSY cooler ring now under construction at Jülich. The main activities of experiments requiring secondary beams (kaons, muons, hyperons, antiprotons) are recommended to take place at CERN's SPS and LEAR.

Physics with electromagnetic probes gets a boost with the new facilities at Mainz, Bonn and Darmstadt, the performances of which allow an optimum study of microscopic nuclear structure and, to some extent, mesonic degrees of freedom. For research programmes requiring high intensity $\mathrm{GeV}$ electrons, the use of CEBAF ( $4 \mathrm{GeV}$ ) now under construction in the USA is encouraged. Consideration of the high energy machine singled out in the French longrange plan for the investigation of quark degrees of freedom was deferred until these lower energy facilities start to produce physics.

In the United Kingdom, the Nuclear Physics Board of the SERC has been reviewing its future activities (3) and foresees the main part of its programme as being concerned with the continuing study of the microscopic structure of nuclei, with beams of heavy ions of energies close to or just above the Coulomb barrier. In the medium term, this type of work mainly requires improved instrumentation for the detection of reaction fragments and for highmultiplicity gamma detection (EUROGAM, EUROBALL).

In the longer term, physicists in the UK envisage the development of a European facility for the production of beams of a wide variety of radioactive ions. Such a facility would be based on generating these ions by spallation using the high energy proton beam from the ISIS spallation neutron source at the Rutherford Laboratory.

The UK's involvement in electromagnetic physics is seen to lie mainly in the study of nucleonic and subnucleonic degrees of freedom of nuclei, with experiments that exploit the Mainz microtron and photon tagger.

In The Netherlands, detailed planning for the long-range future is presently underway (4). The directions that seem to be emerging correspond quite closely to the ones discussed in the French report. Physics with both heavy ion and electron probes will take place in the short term at two new national facilities: AGOR (tens of MeV per nucleon heavy ions), and AMPS ( $800 \mathrm{MeV}$ electrons with a large duty cycle). For the longer range it is envisaged, as discussed in the French planning document, that the most interesting physics will require the application of the high energy accelerators at SIS and CERN for heavy ions, and of a future European high energy accelerator for electrons.

In Italy, the main activities (5) in the near future will concern the investigation of heavy-ion reactions in the $<10$ $\mathrm{MeV}$ per nucleon domain, exploiting the facilities of the Catania and Legnaro centres; an extension to the $1 \mathrm{GeV}$ per nucleon regime, with a facility similar to SIS, is envisaged. The study of nuclei with leptonic probes, aiming at the study of resonances in free and bound nucleons, will concentrate on the beam of monoenergetic photons ( $<1.5 \mathrm{GeV}$ ) that can be produced by electron light scattering at the European Synchrotron Radiation Facility (ESRF) in Grenoble.

At the same time, the Italian Istituto 
Nazionale di Fisica Nucleare (INFN) envisages a major future activity centred on the study of mesonic and quark degrees of freedom of nuclei. In the medium term, this type of research is directed towards the CEBAF facility ( $4 \mathrm{GeV}$ high duty cycle electron accelerator) in the USA. In the longer term, for the study of quark degrees of freedom, the INFN has expressed strong interest in the high energy European electron facility that is discussed in detail in the French report.

\section{European Collaboration}

When looking at nuclear physics in Europe, the field at first sight gives the impression of being largely dominated by national, uncoordinated activities. Funding comes from national agencies; accelerator centres are built and operated by individual nations; decisions concerning the future are mainly taken on a national basis.

At the same time, however, the experimental research itself takes place on a very much European scale. The accelerators situated in Europe are fairly complementary in their performance so experiments must often be done at a foreign laboratory in response to the physics goals - which dictate the use of the most suitable accelerator. Consequently, many research groups are composed of physicists from different countries. The resources of the national laboratories, and the beam time, are attributed to these groups solely on the basis of the merits of the proposed experiment, as evaluated by a programme advisory committee which is almost always international in character.

This international collaboration in most cases takes place on an informal basis, without backing by contracts or agreements between agencies, and is dictated only by the common interests of the researchers who pursue common scientific goals. De facto, European collaboration in nuclear physics is very strong - but it has a low visibility.

It is clear that this mode of operation of nuclear physics will need to be modified in the future. As research projects assume a more complex and larger scale, formal agreements for multinational development are needed. This applies to both the construction and exploitation of apparati needed for the experiments of the various European groups, and, even more so, to the consideration of new facilities like accelerators that will increasingly have a European scale. To cite a few examples that presently are being discussed:

- EUROBALL, the ambulatory, multielement, large solid angle photon detec-

tor, to be used at various heavy-ion facilities:

- the $\mathrm{Pb}$-injector at CERN that should provide relativistic heavy ion beams;

- a future high energy, high duty-cycle European electron accelerator.

Any such project requires that the many steps needed, from the concept and initial planning to proposal and realization, must be taken in a common, European framework.

\section{NuPECC}

NuPECC, the Nuclear Physics European Coordination Committee, a body composed of European physicists has recently been set up to advance planning and similar activities in nuclear physics. This body, with its members nominated initially by the various national funding agencies or physical societies, aims to:

- strengthen European collaboration; - optimize the use of existing national facilities, facilitate the pooling of resources and encourage the development of new instrumentation;

- act as an advisor if requested.

The first steps to improve communication within the European nuclear physics community have been taken. An inventory of facilities has been made, and the structure and editorial board for a news bulletin "Nuclear Physics News", to be edited together with the Board of the Nuclear Physics Division of the European Physical Society, were established earlier this year.

\section{A Nuclear Theory Centre}

The French audit of nuclear physics by members of the Académie des Sciences (see above) recommended the setting up of a nuclear theory centre to help small, sub-critical groups stay in the mainstream, especially in medium energy nuclear physics. A 1988 report of the state of nuclear theory in the USA by a panel of the Nuclear Science Advisory Committee reached a similar conclusion in recommending the creation of at least one theory institute in the United States.

While little has been done so far at the European level to follow up on the idea, the US institute is now in business following acceptance by the Department of Energy of a proposal made by a consortium based on the University of Washington, Seattle. The first 3-5 month programme, on quarks in nuclei, intended for 10-15 scientists at the advanced postdoc level started in March
It is clear that a body such as NUPECC would work best within the framework of established European collaborations in science. The common interests of NuPECC and the European Science Foundation (ESF) in Strasbourg have meant that NuPECC has recently evolved into being an Associated Committee of the ESF, a framework that promises to be very fruitful.

The first action of NUPECC as an associated committee of the ESF relates to the headline of this contribution: NuPECC will be working to bring together a number of outstanding nuclear physicists in Europe in order to elaborate a long-range plan for European nuclear physics.

\section{REFERENCES}

(1) La Physique Nucléaire Fondamentale: Rapport présenté à l'Académie des Sciences par la commission chargée de l'enquête demandée par le Ministre de la Recherche et de la Technologie; G. Charpak, P. Darriulat, M. Goldman and M. Jacob (1990).

(2) Künftige Entwicklung der Grundlagenforschung in der Mittelenergie- und Kernphysik: Bericht des ad-hoc Ausschusses des BMFT; A. Faessler, P. Kienle, K. Kleinknecht, A. Richter, H. Specht and J. Wess (1989).

(3) Memorandum by the Chairman of the Nuclear Structure Committee to the Science and Engineering Research Council (1990).

(4) Report not yet released.

(5) Private communications. with 5-8 international participants.

The plan is to build offices for the institute and to share the facilities of the University's physics department. A permanent Director to replace the interim Director, Professor Ernest Henley is being sought, together with two senior staff. Some funds are available to help meet visitors' salaries - an important element if the institute is to stimulate an increasingly interdisciplinary nuclear physics community, and to help train graduate students and postdoctoral fellows.

Two more programmes - on nuclear astrophysics and on relativistic heavy ion reactions - will be organized in 1990. Those interested in participating should contact Prof. E.N. Henley, Dept. of Physics, FM-15, Univ. of Washington, Seattle, WA 98195;

Tel.: ++1-206-543 27 70;

Fax: $++1-206-6850635$. 